\title{
Étude de l'effet d'échelle sur la cavitation dans les turbomachines hydrauliques
}

\author{
The effect of scale on cavitation \\ in hydraulic turbomachinery
}

\author{
PAR J. BEAUFRËE \\ INGËNIEUR A LA DHRECTION DES ÉTUDES ET RECHERCHES D'ÉLECTRICITÉ DE FRANGE
}

\begin{abstract}
Etade expérimentale des phénomènes de cavitation dans les turbomachines hydrauliques en vue de réduire les marges de sécurité à adopter pour le prototype à partî des résultats obtenus sur modèle réduit.

L'étude théorique de la cavitation indique que les effets d'échelle portent stzr la pression abso. Ine, la pression critique et les termes qui dépendent de la turbulence: couche linite, décollement. Pour une machine $\dot{a}$ axe horizontal, cette étude indique que la similitude de Froude est la similitude qui entraîne l'effet d'échelle le plus faible: celui-ci est pratiquement négligeable, pourvu que la turbulence sur le modèle ne soit pas trop faible, c'est-d-dire que le modelle ne soit pas fait $\dot{a}$ trop petite échelle. L'effet d'échelle qui risque de subsister est d'ailleurs dans le sens de la sécurité : Ia cavitation apparait plus facilement sur le modèle que sur le prolotype, et d'autant plus facilement que la chute d'essai est plus faible.

Les tésultats de cette étude ont ité vérifiés par trois séries d'essais expérimentaux.
\end{abstract}

\begin{abstract}
The author describes an experimental investigation of cavitation phenomena in hydraulic turbomachinery carried out with the intention of reducing the safety factor that has to be allowed on the prototype when using model results.

A theoretical sfud!l shows that scale effects affect the absolute pressure, the eritical pressure and factors such as the boundary layer and breakaway, which depend upon turbulence. The results of this study show that the Froude similitude is the similitude which is associated with the smallest scale effect for horizontal shaft machines and that it can practically be neglected provided that turbulence in the model is not too small, i.e. provided that the scale of the model is not too small. Any remaining scale effects make for greater safety since cabitation occurs more easily in the model than in the prototype, especially since the model head is smaller.

The results of this study were checked by means of three series of experimental tests.
\end{abstract}

L'étude de l'effet d'échelle sur la cavitation dans les turbomachines hydrauliques a un but industriel précis: une installation hydroélectrique coûtera d'autant moins cher que la turbine aura une vitesse de rotation plus grande et une hauteur d'aspiration plus élevée. Mais les constructeurs sont limités dans les valeurs à donner à cette vitesse de rotation et à cette hauteur d'aspiration par les dangers de cavitation, contre lesquels ils prennent une marge de sécurité suffisante. Il s'agit de réduire cette conteuse marge de sécurité en plaçant le point de fonctionnement de la turbine très près du seuil de cavitation. Il faut donc définir et étudier avec soin ce seuil et cette étude ne peut se faire économiquement que sur modèle réduit. Or, la cavitation est un phénomène qui dépend d'un grand nombre de paramètres dont peu sont reproductibles en similitude; il s'introduit donc un effet d'échelle, qui dépend de la similitude choisie. Nous nous proposons de déterminer la meilleure similitude à utiliser pour les études de cavitation de turbomachines hydrauliques sur modèle réduit et d'étudier les variations de l'effet d'échelle dans les différentes similitudes déterminées par la variation de l'échelle des chutes (1).

Nous allons tout d'abord étudier la formation

(1) Voir : The selection of length and head scales for cavitation tests, par Pierre Danel et Jacques Duront, 59-A-40, A.S.M.E. 

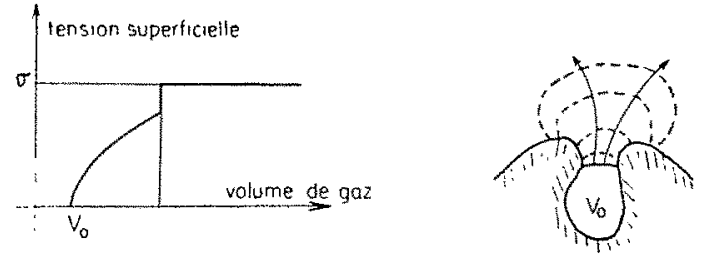

Fia. 1

Grossissement d'un noyau piégé.

de bulles et de cavilés sur les pales des turbomachines, l'effel d'echelle sur cette formation, et nous comparerons les résultats de l'analyse avec les résultats des essais effectués sur des turbines Kaplan à axe horizontal.

L'eau ulilisée dans les chutes industrielles et dans les stations d'essais de modèles réduits contient en général un grand nombre d'impuretés et en particulier de l'air dissous en quantité notable (jusqu'à $25 \mathrm{mg}$ /litre). Quand cette eau est soumise à une pression décroissante, il se produit une bullition gazeuse quand la pression atteint la pression de saturation et une vaporisation quand la pression atteint la pression de vapeur dans les conditions de l'expérience. Ia cavitation est la composition de ces deux phénomènes. Comme tous les phénomènes de changement d'état, elle se produit à partir de germes qui sont ici des petits noyaux d'air. Si la nécessité de la présence de ces noyaux est indiscutable, il faut faire une hypothèse sur leur nature et leur stabilité. Nous supposons que ce sont des noyaux piégés dans des crevasses de petites impurctés solides, de telle sorte que la surface de séparation air-eau soit tres faible. En effet, avec un noyau sphérique de volume équivalent au noyau piégé, il faut des tensions considérables pour que la cavitation puisse apparaître, ce qui est contraire aux résultats de l'expérience. Nous supposons, en outre, que le noyau est initialement en équilibre et que l'effet de la tension superficielle est alors nul. S'il n'en était pas ainsi, la pression dans le noyau serait supérieure à la pression dans le liquide, l'air difluserait et le noyau finirait par disparaître. Quand la pression atteint la pression de saturation ou la pression de vapeur, le noyau grossit par ébullition et vaporisation et il s'ćchappe de la crevasse. Nous sommes amenés à donner une loi de variation de la tension superficielle quand le noyau grossit dans la cavité, loi qui doit marquer une discontinuité quand la bulle s'échappe. Nous pensons qu'elle est de la forme indiquée sur la figure 1, mais cette hypothèse n'a pas encore été vérifiée.

L'insuffisance ou l'absence des noyaux pourra entrainer un retard à la cavitation par surchauffe ou sursaturation de l'eau. Nous appellerons « eau normale » une eau qui contient des noyaux suffisamment nombreux et gros pour que la pression critique d'apparition de la cavitation ne dépende que de la température et de la pression de dissolution de l'air dans l'eau d'expérience, le temps de passage des noyaux dans la zone de basse pression étant suffisant.

On peut considérer que la cavitation devient visible quand les noyaux, qui ont une taille ini-
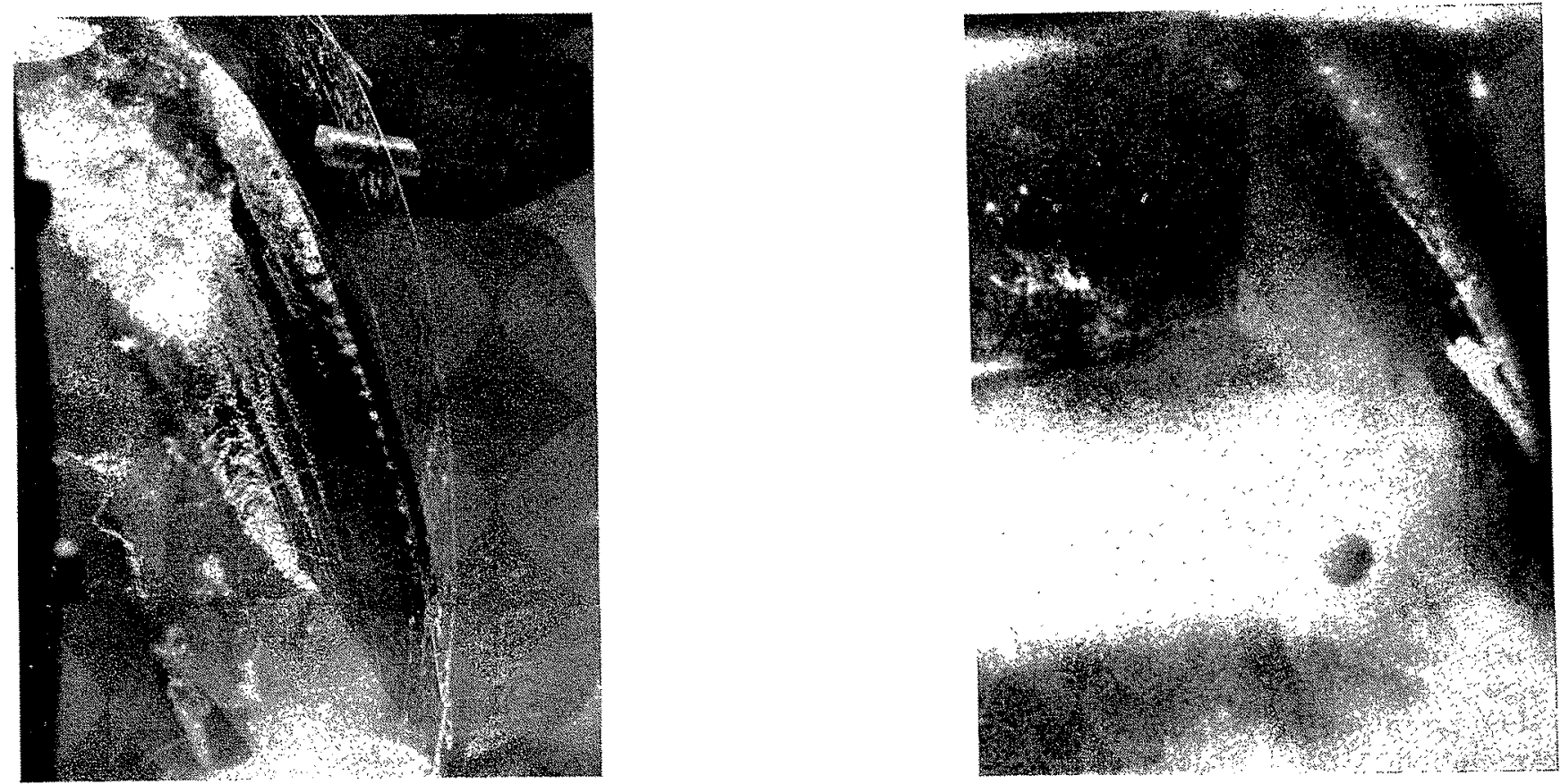

Fig. 2

Diflchentes formes de cavitation. 
tiale voisine de $10^{-4} \mathrm{~cm}$, ont grossi environ 500 fois. Mais en réalité, sur les pales des turbines, il n'apparaît pas que des bulles obtenues par simple grossissement des noyaux : la cavitation se manifeste aussi par des décollements, des cavités périodiques, des petits vortex (fig. 2).

L'état de l'écoulement dans une turbine est caractérisé par le coefficient classique de Thoma:

$$
\sigma_{\mathrm{T}}=\frac{p_{\mathrm{st}}--p_{\mathrm{v}}}{h}
$$

$p_{\mathrm{st}}=$ pression statique au point considéré,

$p_{\mathrm{v}}=$ pression de vapeur,

$h=$ chute d'essai.

Ceci revient à comparer en un point la pression calculée à partir de la pression statique à l'aval de la turbine à la pression critique supposće égale à la pression de vapeur. En réalité, la cavitation sera caractérisce par le coefficient :

$$
\sigma=\frac{p-p_{c r}}{h}
$$

$p=$ pression absolue au point considéré;

$p_{\mathrm{er}}=$ pression critique;

$h=$ chute d'essai.

La cavitation apparaît pour $\sigma=0$.

Nous allons étudier l'effet d'échelle sur la pression absolue $p$ et la pression critique $p_{\mathrm{cr}}$, et comme, sur modèle réduit, nous pouvons choisir arbitrairement l'échelle des chutes, nous allons évaluer les variations de l'effet d'échelle en fonction de l'échelle des chutes.

\section{EFFET D'ECHELLE SUR LA PRESSION ABSOLUE}

La pression en un point de l'extrados d'une pale s'écrit, en appliquant le théorème de Bernoulli entre ce point et l'entrée du diffuseur :

$$
\begin{aligned}
& \frac{\mathrm{C}_{2}{ }^{2}}{2 g}+\frac{p}{\bar{\omega}}+z=\frac{\mathrm{V}_{0}{ }^{2}}{2 g} \\
& +\left(h_{\mathrm{av}}-z-\eta_{\mathrm{d}} \frac{\mathrm{V}_{0}{ }^{2}}{2 g}+\zeta_{\mathrm{d}}\right)+z+\zeta_{\mathrm{Il}}
\end{aligned}
$$

$\mathrm{C}_{2}=$ vitesse absolue sur l'extrados,

$p=$ pression absolue cherchée,

$\mathrm{V}_{0}=$ vitesse à l'entrée du diffuseur,

$h_{a r}=$ pression statique à l'aval du diffuseur, prise sur l'axe,

$z=$ cote du point étudié par rapport à l'axe,

$\eta_{\mathrm{d}}=$ rendement du diffuseur,

$\zeta_{d}=$ pertes de charge dans le diffuseur,

$\zeta_{\mathrm{I}}=$ pertes de charge entre le point étudié et l'entrée du diffuseur.

Le triangle des vitesses à la sortie de la roue donne :

$$
\frac{\mathrm{C}_{2}{ }^{2}}{2 g}=\frac{u \mathrm{C}_{2_{u}}}{g}-\frac{u^{2}-\mathrm{W}_{2}{ }^{2}}{2 g}
$$

$u=$ vitesse d'entrainement;

$\mathrm{C}_{2 u}=$ composante de $\overrightarrow{\mathrm{C}_{2}}$ sur $\vec{u}$;

$\mathrm{W}_{2}=$ vitesse relative.
On en déduit :

$$
\begin{aligned}
p=h_{\mathrm{ar}}-z+ & \left(1-r_{\mathrm{id}}\right) \frac{\mathrm{V}_{\mathrm{u}}{ }^{2}}{2 g} \\
& +\frac{u^{2}-\mathrm{W}_{2}^{2}}{2 g}-\frac{u \mathrm{C}_{\mathrm{g}_{H}}}{g}+\zeta_{\mathrm{d}}+\zeta_{\mathrm{IR}}
\end{aligned}
$$

L'effet d'échelle est caractérisé par les variations de $p / h$ avec $h$.

Les termes qui risquent de subir un effet d'échelle sont :

$$
\frac{z}{h} \quad \frac{\zeta_{n}}{h} \quad \tau_{t a} \frac{V_{n}^{2}}{2 g h} \quad \frac{\mathrm{W}_{2}^{2}}{2 g h}
$$

Effet d'échelle sur $W_{2}^{2} / 2 g h:$ pour calculer la vitesse relative exacte en un point d'une pale, nons utilisons des grilles de profils obtenues par des coupes cylindriques développées de la turbine. Nous remplaçons les profils par des lignes de sources de courant el de tourbillons élémentaires qui induisent, au point considéré, des vitesses dérivant d'un potentiel. L'étude du potentiel créé par ces irrégularités et par le courant montre que le terme $\mathrm{W}_{2}{ }^{2} / 2$ gh est rigoureusement constant el ne subit pas d'effet d'échelle. Il faut noter que, dans ce calcul de la vitesse relative, nous avons supposé que les vitesses radiales étaient nulles.

Effel d'échelle sur les tormes:

$$
\frac{z}{h} \quad \text { et } \quad \eta_{d} \frac{V_{0}^{2}}{2 g h}-\frac{\zeta_{d}}{h}
$$


ces termes donnent la valeur de la pression statique au point considéré. Le terme :

$$
\eta_{\mathrm{d}} \frac{\mathrm{V}_{0}^{2}}{2 g h}-\frac{\zeta_{\mathrm{d}}}{h}
$$

traduit la récupération de pression du diffuseur. Toutes les études effectuées jusqu'à maintenant sur les diffuseurs ont été faites en utilisant l'air comme fluide. Elles indiquent que la similitude de Reynolds est la similitude qui n'introduit pas d'effet d'échelle, mais qu'en fait il suffit d'avoir un nombre de Reynolds suffisant pour que les termes :

$$
\eta_{\mathrm{d}} \frac{\mathrm{V}_{0}^{2}}{2 g h}-\frac{\zeta_{\mathrm{d}}}{h}
$$

restent constants quand $h$ varie. Pour que la ré- partilion de la cavilalion soil géométriquement semblable autour de la roue modèle et de la roue prototype, il faut opérer en similitude de Froude pour avoir $\left(z_{p} / h_{p}\right)=\left(z_{m} / h_{m}\right)$. Pour toute autre similitude, il y aura distorsion : pour les très fortes chutes, la cavitation sera la même tout autour de la roue, tandis que, pour les très failes chutes, elle sera localisèe dans la partie supérieure.

La pression que nous avons calculée dépend des termes dynamiques et des termes statiques. Il faut lui ajouter les fluctuations locales dues à la turbulence, aux caractéristiques de la couche limite et à la formation de petits vortex. Pour que la similitude soit rigoureuse, il faut que les nombres de Froude et de Reynolds soient les mêmes sur le modèle et sur le prototype; il suffira en réalité d'opérer en similitude de Froude avec un nombre de Reynolds assez élevé.

\section{EFFET D'ECHELLE SUR LA PRESSION CRITIQUE}

Nous avons vu que le seuil de cavitation pouvait être déterminé par un grossissement fixé des noyaux. Nous supposerons que les noyaux sont identiques à l'entrée du modèle et du prototype; l'effet d'échelle portera donc sur le temps de passage des noyaux dans la zone de basse pression, sur la forme de cette zone, et sur la valeur de la dépression maximum. Il est difficile de faire une étude quantitative, surtout en ce qui concerne les caractéristiques de la zone de basse pression, car elles dépendent de leffet d'échelle dû à l'écart de la similitude choisie avec les similitudes de Froude et de Reynolds. On peut conclure seulement que plus la chute d'essai est faible, plus la cavitation est développée, car la couche limite est relativement trop épaisse, et plus elle est gazeuse, car le temps de passage des noyaux dans la zone de basse pression est plus long (cette comparaison se fait pour des conditions de pression semblables). Nous chercherons expérimentalement une loi de variation de la pression critique avec la chute d'essai.

L'étude de la pression absolue et de la pression critique nous permet de prévoir l'effet d'échelle sur le lieu de cavitation et sur le rendement; quand, à partir d'un point de fonctionnement donné, on diminue la pression à l'aval de la roue, la cavitation se répartit d'autant plus vite autour de la roue que la chute est plus élevée, et la chute de rendement est d'autant plus rapide.

\section{EFFET D'ÉCHELLE SUR LE COEFFICIENT DE THOMA}

Bien que ce coefficient traduise mal, comme nous l'avons vu, l'état de l'écoulement dans une turbine, il est couramment employé sur les platesformes d'essais à cause de sa simplicité. L'étude de l'effet d'échelle subi par les termes $p / h$ et $p_{y} / h$ indique que quand la chute d'essai croit, le $\sigma_{\mathrm{Ta}}$ d'apparition de la cavitation décrôt et que le $\sigma_{\text {Ter }}$ critique correspondant à une chute de rendement de $1 \%$ croît. Mais il nous est impossible de donner une loi de variation de ces $\sigma_{\mathrm{T}}$, car nous ne pouvons pas chiffrer le rôle joué par les caractéristiques de l'eau (tension superficielle, nature des noyaux). Ces lois de variations seront déterminées expérimentalement.

De l'analyse de l'effet d'échelle, nous pouvons conclure que la meilleure similitude à utiliser dans les essais de cavitation de turbomachines a axe horizontal est la similitude de Froude, pourvu que le modèle ne soit pas réduit à une échelle trop petite. Si le nombre de Reynolds est de l'ordre de $10^{\circ}$ très près du bord d'attaque des pales, l'effet d'échelle est très faible, et il est 
dans le sens de la sécurité. En effet, l'ulilisation de la similitude de Froude impose en général des chutes d'essai faibles, pour lesquelles la cavitation apparait facilement : alors que, dans les machines industrielles, l'ébullition gazcuse est très réduite, elle apparait facilement sur les modèles réduits fonctionnant sous une basse chute.

\section{VERIFICATION EXPERIMENTALE}

Les essais expérimentaux ont surtout pour but d'étudier la variation de l'effet d'échelle avec l'échelle des chutes, et de déterminer la loi de variation des $\sigma$ de Thoma correspondant au seuil de cavitation et à la chute de rendement de

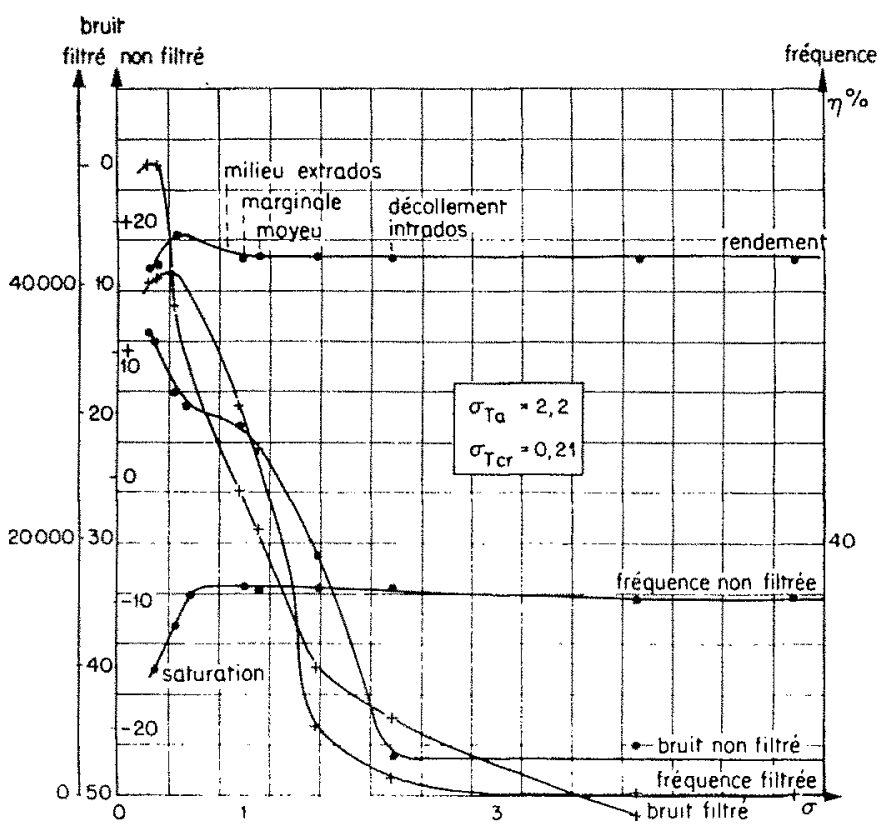

Fig. 3

Détermination des orn et oTer.

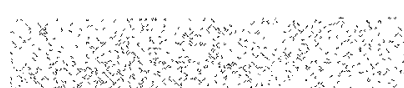

$1 \%$. En outre, une tentative a été faite pour comparer un prototype et son modèle réduit.

Pour déterminer l'état de cavitation, nous avons utilisé, sur la station d'essais de turbines de Chatou :

- Les grandeurs énergétiques :

- mesure du rendement.

- Les pressions acoustiques :

- observation des impulsions à l'oscilloscope;

- enregistrement d'oscillogrammes;

- mesure du bruit moyen;

- mesure de la pseudo-fréquence des impulsions.

- Les observations optiques :

- stroboscopie;

- photographie $\left(10^{-6}\right.$ seconde);

- cinéma ultra-rapide (3000 images/s.).

Tous ces procédés nous ont permis de trouver les courbes de rendement de bruil et de fré-
PLANCHE 53 bIS

Thlodos:
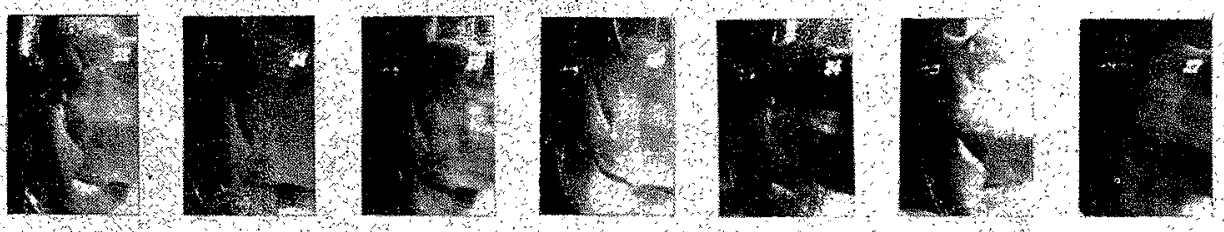

029003

0,4

0.55

0.7

0.99 $112 \quad a$
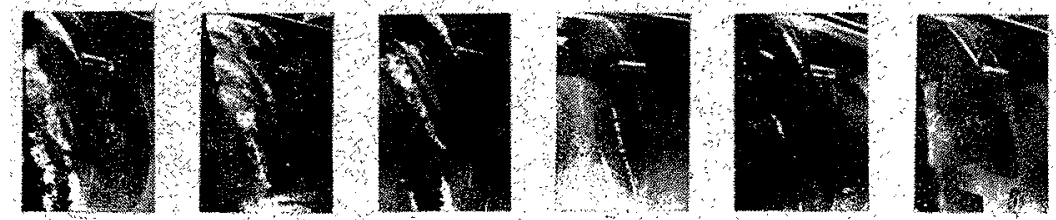

Extrados 


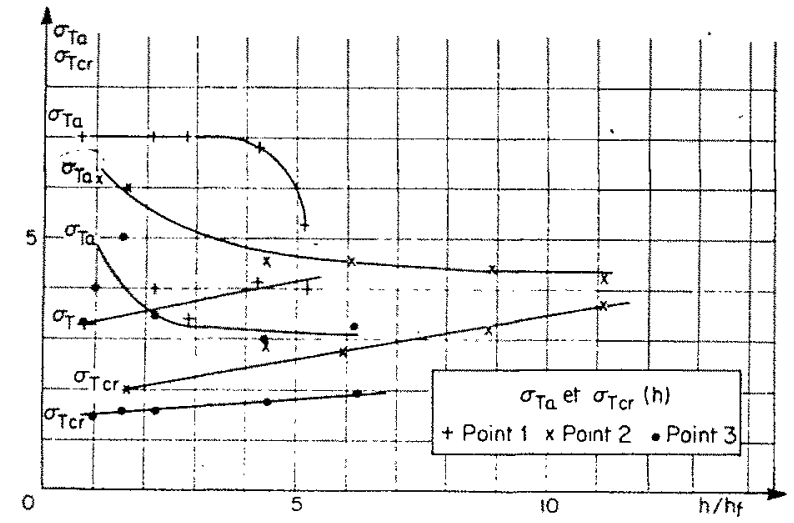

Fia. 4

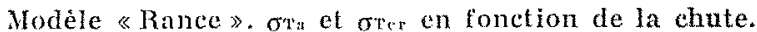

quence en fonction du o de Thoma. En régime non cavitant, ces grandeurs sont constantes, mais elles varient rapidement dès qu'apparaissent les premières bulles de cavitation. En accord avec les observations optiques (fig. 3), nous pouvons déterminer, grâce à ces courbes, le $\sigma_{\mathrm{TA}}$ d'apparition de la cavitation avec une précision comprise entre 5 et $10 \%$ suivant les points de fonctionnement de la turbine. Le $\sigma_{\mathrm{T}}$ critique est déterminé uniquement grâce aux mesures énergétiques, et la précision obtenue est de l'ordre de $5 \%$. Mais, dans cerlains cas, il pent y avoir ambiguité sur sa détermination. Pour lever cette ambiguité, il faut s'assurer que les essais de cavitation sont poussés jusqu'à un stade tel que le rendement ait très nettement diminué.

Les essais ont été effectués sur deux modèles réduits, les modèles « Rance » et «Cambeyrac » de turbines destinées à équiper des groupes bulbes à axe horizontal. Il a, en outre, été possible de faire des essais de comparaison entre le modèle et le prototype « Cambeyrac s,

L'étude effectuée sur le modèle « Rance»a permis d'utiliser une gamme de chutes d'essai assez étendue, et nous sommes même parvenus à réaliser une chute d'essai égale à la chute industrielle. Les résultats obtenus sont en accord avec ceux de l'analyse : quand la chute d'essai croit, $\sigma_{\mathrm{Ter}}$ croît et $\sigma_{\mathrm{Tn}}$ décroît (fig. 4).

L'analyse de l'effet d'échelle nous a montré que le terme $p / h$ reste sensiblement constant quand la chute d'essai varie. L'effet d'échelle sur le $\sigma_{\mathrm{T} n}$ porte done sur le terme $p_{\mathrm{v}} / h$. Si la pression critique est supposée constante et égale à la pression de vapeur, la variation de $\sigma_{\mathrm{wa}}$ sera hyperbolique. Or, cette pression critique varie, et les essais ont montré que, pour obtenir une bonne représentation du phénomène, il fallait corriger la forme hyperbolique par une exponentielle:

$$
\sigma_{\mathrm{T} \mathrm{a}}=\mathrm{A}+\frac{\mathrm{B}}{h / h_{\mathrm{F}}}+\mathrm{C} e^{-\mathrm{v} h / h_{\mathrm{F}}}
$$

$h=$ chute d'essai,

$h_{\mathrm{F}}=$ chute d'essai en similitude de Froude, A B C D $=$ constantes qui dépendent des caractéristiques de la turbine et du point de fonctionnement étudié.

Cette équation nous donne une première méthode pour calculer la pression critique, en utilisant le $\sigma$ idéal défini plus haut. Ce $\sigma$ est nul au seuil de cavitation, et nous pouvons l'écrire, en posant $p_{\mathrm{cr}}=k p_{\mathrm{v}}$ :

$$
\sigma_{\mathrm{H}}=\frac{p_{\mathrm{abs}}-p_{\mathrm{cr}}}{h}=\frac{p_{\mathrm{abs}}-k(h) p_{\mathrm{v}}}{h}
$$

En première approximation, nous pouvons écrire, pour le seuil de cavitation :

$$
\begin{aligned}
\sigma_{\mathrm{T}} & =\frac{p-p_{\mathrm{v}}}{h}=\mathrm{A}+\frac{\mathrm{B}}{h / h_{\mathrm{F}}}+\mathrm{C} e^{-\mathrm{D} h_{h} / h_{\mathrm{F}}} \\
& =\frac{p-k p_{\mathrm{v}}+k p_{\mathrm{v}}-p_{\mathrm{v}}}{h}=\sigma_{\mathrm{H} \Omega}+\frac{p_{\mathrm{v}}}{h}(k-1)
\end{aligned}
$$

Comme $\sigma_{\mathrm{Ha}}=0$, nous avons :

$$
p_{\mathrm{cr}}=p_{\mathrm{r}}+\mathrm{B} h_{\mathrm{F}}+\mathrm{A} h+\mathrm{C} h e^{-\mathrm{D} h / h_{\mathrm{F}}}
$$

Nous pouvons, d'autre part, calculer la pression critique par des mesures de dimensions de bulles en des points de fonctionnement très voisins du seuil de cavitation. Une généralisation de l'équation de Lord Rayleigh nous donne la pression dans la bulle:

$$
p=p_{\mathrm{r}}+p_{\mathrm{x}}=?\left(\mathrm{RR}^{\prime \prime}+3 / 2 \mathrm{R}^{2}+2 \sigma / \mathrm{R}\right) g+p(t)
$$

$p_{\mathrm{g}}==$ pression partielle de gaz dans la bulle;

$$
\rho=\text { densité de l'eau; }
$$

$R(t)=$ rayon de la bulle, fonction du temps. Cette fonction est déterminée par des mesures faites sur des films pris à la caméra ultra-rapide;

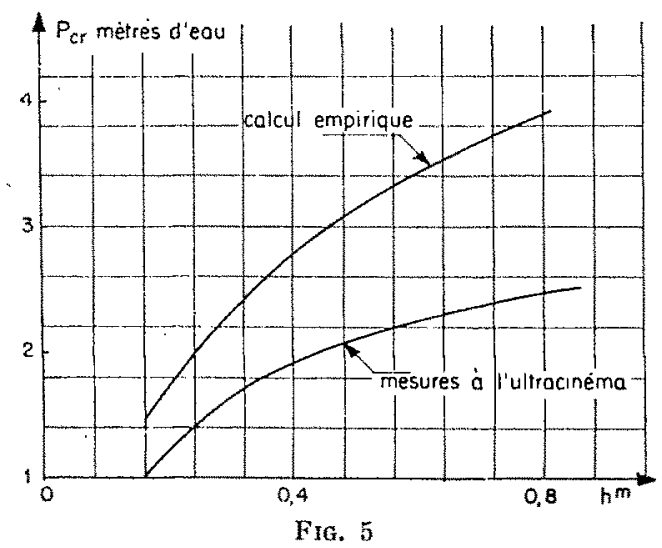

Pression critique en fonction de la chute d'essai. 
$\sigma=$ tension superficielle;

$p(t)=\begin{gathered}\text { pression statique au centre de la bulle } \\ \text { s'il n'y avait pas cavitation. }\end{gathered}$

Les mesures à l'ultra-cinéma sont encore, hélas, très imprécises, et nous n'obtenons que des résultats approchés.

Les résultats de ces deux modes de détermination de la pression eritique indiquent que la pression critique croît avec la chute d'essai, et que le coefficient $k\left(p_{\text {cr }}=k p_{v}\right)$ est bien supérieur à 1 (de l'ordre de 4 à 5) (fig. 5).

D'autre part, les observations à l'ultra-cinéma montrent que la cavitation est d'autant plus gazeuse que la chute d'essai est plus faible.

Les essais effectués sur le modèle «Cambeyrac » sont beaucoup moins significatifs que les précédents (fig. 6); en effet, pour les points de fonctionnement étudiés, la gamme de chutes d'essais est très réduite. Ces points nous ont été imposés, car ils étaient les seuls à pouvoir être étudiés sur le prototype, en vue d'une comparaison. Les résultats concordent néanmoins avec ceux de l'analyse et ceux du modèle "Rance», sauf en ce qui concerne une courbe $\sigma_{\mathrm{Ta}}(h)$.

Il a été possible, pour quelques points de fonelionnement en turbine inversée, de faire varier le $\sigma_{\mathrm{T}}$ sur le prototype «Cambeyrac ». Mais ces variations sont faibles, et les parties observa-

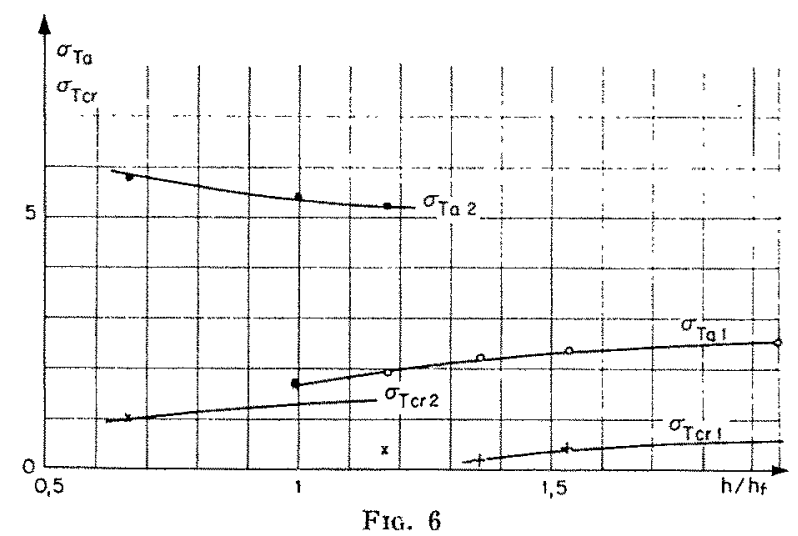

Modèle "Cambeyrac», or" et orer en fonction de la chute.

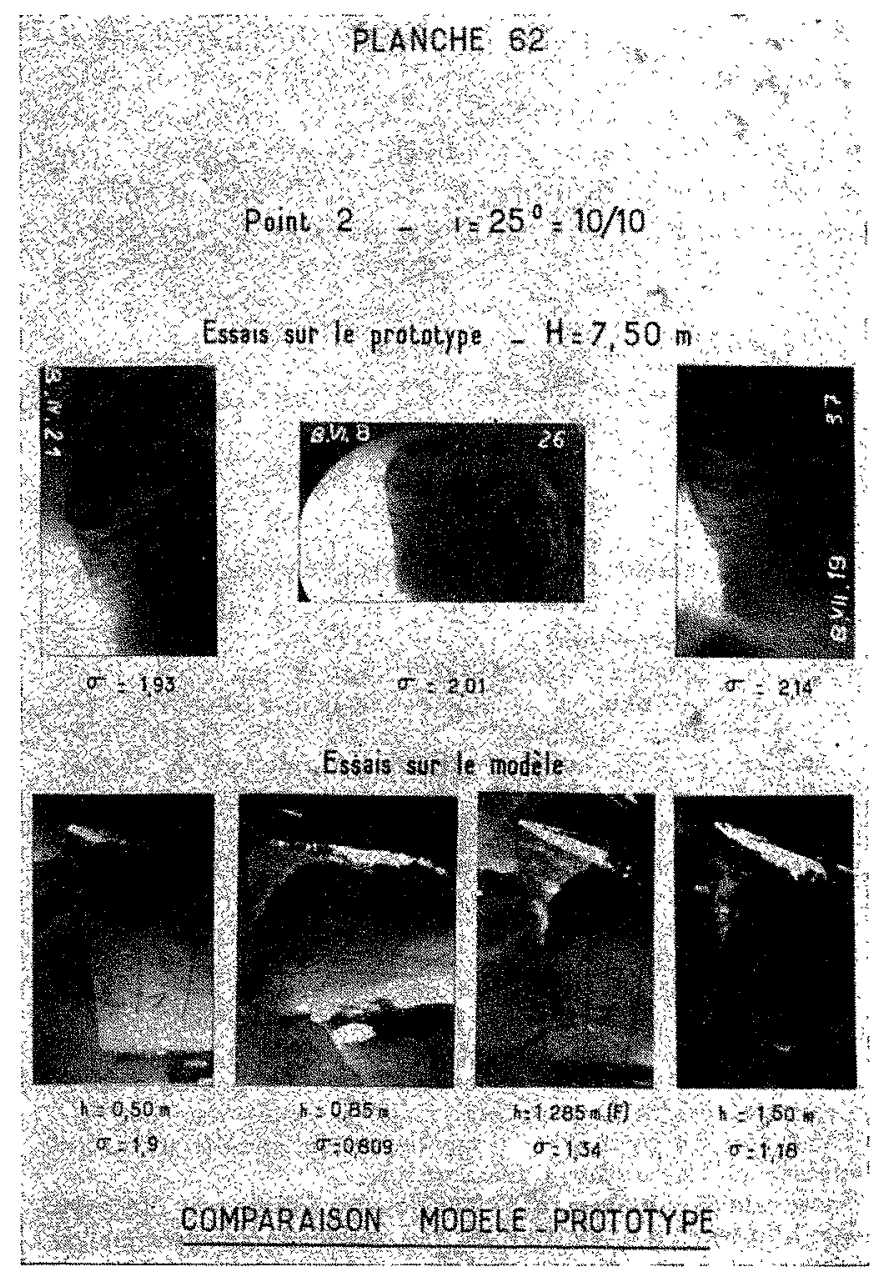

Fra. 7

bles de la turbine très réduites: les observations optiques se réduisent au bord d'attaque intrados et congé de raccordement de la pale au moyeu à l'extrados, côté bord d'attaque. La comparaison entre le modèle et le prototype s'est limitée au décollement à partir du bord d'attaque, côté intrados (fig. 7). Or, ce décollement est très sensible à la moindre variation d'angle d'attaque, et cetle variation masque l'effet d'échelle. Les mesures acoustiques n'ont pas donné de résultats à cause de l'importance des bruits d'origine mécanique.

\section{CONCLUSION}

Il est difficile de pouvoir formuler des résultats numériques an sujet de l'effet d'échelle, car nous nous heurtons d'une part à une imposssibilité de mettre sous forme mathématique les pro. priétés de l'eau, les effets des noyaux et de la turbulence, et d'autre part, aux difficultés des essais de cavitation : il est très délical de fixer avec une grande précision le org d'apparition de la cavitation nuisible. Nous pouvons cependant affirmer que quand la chute d'essai croit, le rirn décroit, tandis que le $\sigma_{\text {Ter }}$ croît. En ce qui concerne la détermination de la meilleure simili- 
tude à utiliser pour les essais de cavitation de machines à axe horizontal, l'expérience ne nous a pas encore fourni de résultats valables, mais l'analyse de l'effet d'échelle nous indique que, pourvu que le nombre de Reynolds soit suffisamment grand, il y a intérêt à utiliser la similitude de Froude. D'autre part, les études expérimentales, en particulier l'ultra-cinéma, ont mis en évidence l'importance du rôle joué par la cavitation gazeuse, surtout pour les faibles chutes d'essai.

\section{I C USSION}

Président : M. Ligovzat

M. le Président remercie M. Beaufrère de son intéressant exposé.

M. Lugrez demande :

$1^{\circ} \mathrm{Si}$ les mesures acoustiques dont a parlé M. Brauprère ont mis en évidence des bruits de fréquences déterminées, et, dans ce cas, s'il a été possible de relier ces fréquences à divers paramètres de l'essai; $2^{\circ}$ S'il a été possible de retrouver la fréquence de $30000 \mathrm{~Hz}$ sur la machine industrielle.

M. BEAufrìne indique qu'au cours des divers essais, l'analyse de la fréquence du bruit de cavitation montre un maximum du niveau de bruit pour des fréquences de l'ordre de $30000 \mathrm{~Hz}$; elle a mis en outre en évidence un nivean de bruit notable pour des réquences de l'ordre de $100000 \mathrm{~Hz}$, fréquences qui sont très caractéristiques de la cavitation. Elles peuvent être captées sur le modèle réduit, car alors le bruit est peu amorti par la couche d'eau qui est mince, mais elles ne peuvent pas être utilisées sur un prototype pour lequel la couche d'ean est épaisse et l'absorption très grande.

Pour la fréquence de $30000 \mathrm{~Hz}$, des essais ont été faits sur le prototype de Cambeyrac, mais les bruits mécaniques étaient très supérieurs au bruit de cavitation, qui n'a pas pu être caractérisé.

M. le Président demande comment se forment les premières bulles « piégées » à la surface de contact de l'eau.
M. Beaufrène énonce l'hypothèse qui permet d'expliquer la formation des bulles : la cavitation se produit à partir de petits noyaux qui sont des volumes d'air piégés dans des crevasses d'impuretés solides et qui sont en équilibre au sein de la masse liquide : lorsque ces noyaux sont soumis à une pression décroissante, ils grossissent, se développent et forment les bulles de ca. vitation. Pour que cette hypothèse soit valable, il faut supposer que la surface de séparation entre le gaz et la masse liquide soit plane, ce qui n'est pas vérifié, ou que la tension superficielle suive une loi qui la fasse passer progressivement de 0 à $\sigma$.

M. Duront remarque que dans le «raccourci \& très condensé du début de son exposé, M. Beaufrère a fait allusion à l'influence du nombre de Reynolds sur le coefficient de récupération de l'aspirateur. La viscosité et par conséquent le nombre de Reynolds peuvent également influencer le champ de vitesse et de pression dans la roue.

M. BEAUFrère mentionne également l'hypothèse de calcul par laquelle on décompose la turbine en turbines élémentaires cylindriques coaxiales, négligeant ainsi les composantes radiales de la vitesse d'écoulement. En fait ces composantes radiales ne créent pas, par elles-mêmes, un effet d'échelle, et sont convenablement reproduites sur le modèle, quelle que soit d'ailleurs l'échelle des chutes (en l'absence de cavitation). 